\title{
An evolutionary ratchet leading to loss of elongation factors in eukaryotes
}

\author{
Gemma C Atkinson 1,2,3* , Anton Kuzmenko ${ }^{1,4}$, Ivan Chicherin ${ }^{4}$, Axel Soosaar ${ }^{1}$, Tanel Tenson ${ }^{1}$, Martin Carr ${ }^{5}$, \\ Piotr Kamenski ${ }^{4}$ and Vasili Hauryliuk $k^{1,2,3}$
}

\begin{abstract}
Background: The GTPase eEF1A is the eukaryotic factor responsible for the essential, universal function of aminoacyl-tRNA delivery to the ribosome. Surprisingly, eEF1A is not universally present in eukaryotes, being replaced by the paralog EFL independently in multiple lineages. The driving force behind this unusually frequent replacement is poorly understood.

Results: Through sequence searching of genomic and EST databases, we find a striking association of eEF1A replacement by EFL and loss of eEF1A's guanine exchange factor, eEF1Ba, suggesting that EFL is able to spontaneously recharge with GTP. Sequence conservation and homology modeling analyses indicate several sequence regions that may be responsible for EFL's lack of requirement for eEF1Ba.

Conclusions: We propose that the unusual pattern of eEF1A, EEF1Ba and EFL presence and absence can be explained by a ratchet-like process: if either eEF1A or eEF1Ba diverges beyond functionality in the presence of $\mathrm{EFL}$, the system is unable to return to the ancestral, eEF1A:eEFBa-driven state.
\end{abstract}

Keywords: eEF1A, EFL, eEF1B, Ribosome, Elongation factor, GTPase, GEF, Molecular evolution, Eukaryotes

\section{Background}

EF1A in eukaryotes (eEF1A) and archaea (aEF1A) is a highly expressed essential GTPase translation factor. Just like its bacterial ortholog, EF-Tu, EF1A delivers aminoacyl-tRNA (aa-tRNA) to the ribosome in complex with GTP during the elongation stage of translation. Accommodation of the aa-tRNA in the ribosomal A site induces GTP hydrolysis by EF1A, releasing the GDP-bound factor from the ribosome [1]. GDP bound to eEF1A needs to be replaced with GTP for the next functional cycle to begin. Some translational GTPases such as the close relative of EF1A, SelB, dissociate GDP rapidly, which leads to spontaneous recharging [2]. However, dissociation of GDP from EF-Tu and EF1A is extremely slow $[3,4]$ and therefore these GTPases require a dedicated guanine exchange factor (GEF) for recharging: EF-Ts in bacteria and EF1B in eukaryotes (eEF1B) and archaea (aEF1B) [5]. Unlike EF-Ts, eEF1B is a multi-subunit protein, with GEF activity residing in the alpha subunit $(\mathrm{eEF} 1 \mathrm{~B} \alpha)$

\footnotetext{
* Correspondence: Gemma.atkinson@ut.ee

${ }^{1}$ University of Tartu, Institute of Technology, Nooruse 1, 50411 Tartu, Estonia

${ }^{2}$ Department of Molecular Biology, Umeå University, Umeå, Sweden

Full list of author information is available at the end of the article
}

$[3,6]$. The crystal structure of the eEF1A:eEF1B $\alpha$ carboxy terminus complex has shed light on the mechanisms of exchange at the molecular level, showing which parts of eEF1A and eEF1B $\alpha$ interact and how this brings about GDP dissociation $[7,8]$.

Besides its role in translation, eEF1A has a variety of additional, "moonlighting" functions. These include actin bundling, nuclear export of aa-tRNAs, proteolysis of misfolded proteins, modulating apoptosis, response to amino acid starvation and viral replication $[9,10]$. Being a universally essential protein in translation, and an accessory protein in a variety of other processes, the discovery of a lack of eEF1A in some eukaryotes was unexpected [11]. In these eEF1A-lacking organisms, another related factor, EFL (for EF1A-like), is present. EFL carries the same domain structure as eEF1A and is presumably functionally equivalent. Most surprising is the broad but discontinuous distribution of EFL in eukaryotes and usually mutual exclusivity with eEF1A $[11,12]$. The pattern of presence and absence has been explained both by horizontal gene transfer (HGT) and co-maintenance and long term co-maintenance followed by lineage sorting (some lineages losing eEF1A and some losing EFL) [12-17]. Given the 
absence of strong support for any specific instance of HGT of EFL, the possibility that the last common ancestor of eukaryotes carried both eEF1A and EFL can not be ruled out [12]. However, this would have required millions of years of functional redundancy in the co-maintenance of eEF1A and EFL without either being lost before the divergence of all eukaryotic groups that carry EFL. Regardless of the mode of descent of EFL, the ancestral state at the last common ancestor of eukaryotes and archaea is likely to be co-presence of eEF1A and eEF1B $\alpha$ and absence of EFL, as aEF1A and aEF1B are found in all archaea, but EFL has never been identified in this domain of life.

Due to a near complete absence of experimental investigations of EFL, the evolutionary mechanisms driving mutual exclusivity of eEF1A and EFL are poorly understood. We hypothesize that the key to this phenomenon lies in the differences in the functional cycle of the two proteins. It was briefly noted [15] that the GEF eEF1B $\alpha$ has not been identified in the genomes of EFL-containing organisms Thalassiosira, Chlamydomonas and Ostreococcus, suggesting that EFL may self-recharge like some other translational GTPases, or that a non-homologous GEF may be involved. With the increasing number of genomes and large scale EST data available for many eukaryotes including those that carry EFL, we have conducted a large-scale survey of EFL, eEF1A and eEF1B $\alpha$ presence and absence across the eukaryotic tree of life. We show a striking association of EFL presence with loss of eEF1B $\alpha$ and eEF1A. We hypothesise a ratchet-like evolutionary process of reduction: if eEF1A or eEF1B $\alpha$ diverges beyond functionality in the presence of EFL, the system is unable to return to the ancestral, eEF1A:eEFB $\alpha$-driven state. Whether EFL loss is similarly irremediable depends on the rate of HGT of this factor.

Motivated by our hypothesis, we set out to test whether EFL can substitute for a loss of eEF1B $\alpha$ in an organism possessing eEF1A gene. Similarly to a previous study that found Diplomena EFL can not substitute for eEF1A in Trypanosoma [18], we find that Monosiga brevicolis EFL is unable to substitute for either double eEF1A and eEF1B $\alpha$ or single eEF1B $\alpha$ deletion in Saccharomyces cerevisiae, thus suggesting an existence of functional barriers to the spread of EFL across eukaryotes.

\section{Results}

Sequence searching of genomic and EST databases shows a striking pattern of presence and absence of EFL, eEF1A and eEF1B $\alpha$ (Figure 1 and Additional file 1: Table S1). Both EFL and eEF1A are present in all major lineages of eukaryotes, and their presence is mostly - but not universally mutually exclusive, as previously reported [11]. Supporting the result of Szabova et al. [18] that experimentally showed eEF1A and EFL can be co-maintained without affecting growth, eEF1A and EFL can be co-maintained in a modest number of organisms (Thalassiosira pseudonana, Guillardia theta, Karenia brevis, Symbiodinium sp. and Ulva prolifera), although these organisms carry no detectable eEF1B $\alpha$. The gut fungus Basidiobolus ranarum has also been found to carry both eEF1A and EFL [19], however in the absence of a genome or EST project to search for eEF1B $\alpha$, it was not included in our survey. This comaintenance may reflect incomplete lineage sorting, or may be because eEF1A is still required for one of its moonlighting functions in these organisms. Importantly, we find the loss of eEF1A is almost universally accompanied by parallel loss of eEF1B $\alpha$, suggesting that EFL functioning does not require the assistance of this GEF (Figure 1 and Additional file 1: Table S1). In some rare cases where eEF1A or eEF1B $\alpha$ are detectable along with $\mathrm{EFL}$, degradation of eEF1A or eEF1B $\alpha$ sequence is apparent, even in functionally important sites; eEF1A appears to be evolving with an apparent loss of selective constraint on the protein sequence in Allomyces macrogynus, Aspergillus niger, Pseudo-nitzschia multiseries, Fragilariopsis cylindrus, Bigelowiella natans, Chlamydomonas reinhardtii and Volvox carteri, while eEF $1 \mathrm{~B} \alpha$ is in the process of decay in Guillardia theta and Pythium ultimum. Thecamonas trahens on the other hand encodes a divergent EFL along with eEF1A and an apparent eEF1B-kinase protein fusion (Figure 1 and Additional file 1: Table S1). Thus, in our snapshot of eukaryotic history, we have captured multiple ongoing cases of gene degradation towards loss. Overall, the association of EFL presence with eEF1A and eEF1B $\alpha$ loss is statistically significant whether the divergent sequences are considered as present or absent $(p<0.00001$ in both cases).

Phylogenetic analysis of the EFL sequences detected in this study gives a tree that is overall similar to other published EFL phylogenies (for example $[13,14,16,20]$ ), although with fewer taxa as we only considered organisms with large scale EST and whole genome data available (Additional file 2: Figure S1A). Even with additional sequences from PCR amplification and sequencing of individual genes, phylogenetic analysis of EFL does not shed much light on the origin and deep evolutionary history of EFL; the deepest branches in the phylogenetic tree lack strong statistical support in ML and/or Bayesian analyses, and the EFL tree can not be rooted reliably due to long branch attraction of divergent sequences to the outgroup [20]. Therefore, the path to EFL replacement of eEF1A (HGT or co-maintenance with differential loss) is hard to determine, as also found with probabilistic models of EFL gain and loss [21]. However, we do see strong bootstrap and posterior probability support for some taxonomic assemblages within the EFL tree, for example Dinophyceae (89\% MLBP (maximum likelihood bootstrap percentage) and 1.0 BIPP (Bayesian inference posterior probability)), Rhodophyta (99\% MLBP, 1.0 BIPP), 


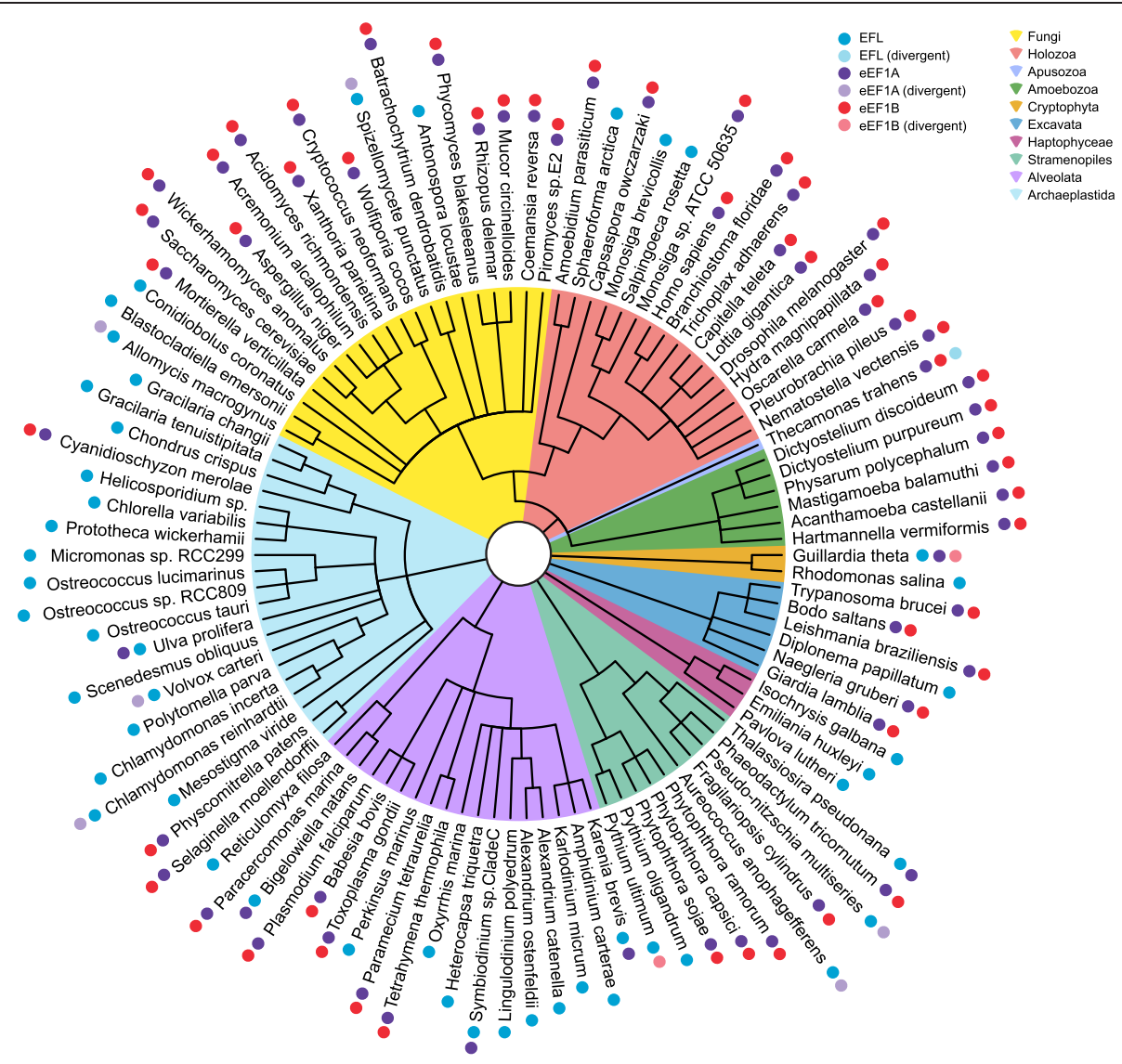

Figure 1 Cladogram showing presence and absence of elongation factors across eukaryotes. The tree summarizes current knowledge of the taxonomic grouping of the species considered here. Polytomies are present where branching order is unknown or contentious. Colored shading behind branches indicates major lineages, as per the color key in the top left. Circles show presence and absence of intact (opaque) or degraded (semi-transparent) elongation factors, with colors indicating factor identity according to the top left key.

fungi, excluding Conidiobolus cornatus (99\% MLBP, 1.0 BIPP), stramenopiles (Heterokontophyta + Oomycetes, 100\% MLBP, 1.0 BIPP) and members of Choanoflagellatea + Ichthyosporea (100\% MLBP, 1.0 BIPP). Dinophyceae and Rhodophyta are all EFL encoding, suggesting that EFL was vertically inherited within these groups. Surprisingly, EST evidence suggests eEF1A has not been completely lost in Dinophyceae, with eEF1A ESTs being detected in Symbiodinium sp. and Karenina brevis (Figure 1 and Additional file 1: Table S1). In the case of Choanoflagellatea, Ichthyosporea, stramenopiles and fungi, some species encode EFL while some encode eEF1A. It is unclear whether co-maintenance and differential loss alone is responsible for this distribution, or whether HGT has been involved. Whatever the source of EFL in these taxa, lineage sorting appears incomplete, with eEF1A sequence relics being detected in some EFL-encoding stramenopiles and fungi. The backbone of the eEF1A tree is poorly resolved and multiple paralogs of eEF1A are apparent (Additional file 2: Figure S1B). Some of these are highly divergent, such as those from Tetrahymena thermophila and Paramecium tetraurelia, which are attracted to the degrading eEF1As in organisms encoding EFL (Additional file 2: Figure S1B).

Comparison of patterns of evolution across sites using a consensus sequence alignment of eEF1A versus EFL (Figure 2) shows differentially conserved sites across all domains (G domain, domain II and domain III), with some such sites clustering together. To determine how the sequence changes affect the structural contacts of EFL, a homology model was made of Chlamydomonas incerta EFL using the X-ray crystal structure of S. cerevisiae eEF1A in complex with eEF1B $\alpha$ as the template (PDB ID 1IJE) [8].

Many of the eEF1A residues that interact with eEF1B $\alpha$ overlap with regions important for nucleotide or aa-tRNA binding (Figure 2). Therefore, it is unsurprising that these multifunctional regions are well conserved in EFL. However, regions of eEF1A that are apparently specialized for $\mathrm{eEF} 1 \mathrm{~B} \alpha$ binding are often very different in EFL. The GTPase (G) domain is overall well conserved, particularly in the nucleotide binding loops, with less conservation 
seen in the exposed loops in between. One of the most striking differences between eEF1A and EFL in this domain is a six amino acid-long strongly differentially conserved patch, Figure 2 coordinates 74-79, (consensus sequences aCTTKA in EFL and DIALWK in eEF1A) that is located in a helix between the G2 and G3 nucleotide binding motifs. The DIALWK motif is part of a loop of eEF1A that directly interacts with eEF1B $\alpha$ through hydrogen bonds (Figure 3A and B) [7]. The conformation of this loop is also stabilized by another strongly differentially conserved site: alignment position 35 (D35 in eEF1A and P35 in EFL, Figure 2). This suggests that the striking sequence differences in these residues are directly related to EFL's apparent lack of requirement for eEF1B $\alpha$. Another differentially conserved residue of this domain that in eEF1A may interact with eEF1B $\alpha$ is in position 106 in Figure 2 (T106 in eEF1A and A106 in EFL, Figure 3A).

Three of the five sequence insertions in EFL relative to eEF1A (Ins1-5, Figure 2) are found in the G domain. Structural alignment of EFL with the structure of eEF1A's bacterial ortholog EF-Tu on the ribosome [22] suggests Ins1 and Ins2 are exposed with no obvious ribosomal interaction partners, but Ins3 extends a helix-loop-helix structural element on the ribosome-binding face (Figure 3C). This insertion is also interesting as it overlaps with a 12 amino acid insertion in opisthokont eEF1A [23]. However, the sequence alignment of these two insertions relative to each other is ambiguous, and thus there is no evidence that the Ins3 insertion is homologous to the animal/fungal insertion. A single conserved amino acid deletion in EFL relative to eEF1A is also apparent, but is found in an exposed loop of eEF1A that is poorly conserved (position 120 in Figure 2).

In domain II, very strong conservation between eEF1A and EFL is seen in the regions that in bacterial EF-Tu form the pocket for accommodating the aminoacyl moiety of aa-tRNA [22] (Figure 2). Differentially conserved sites in this domain are largely in exposed loops, however there are three residues that in eEF1A are positioned to potentially interact with $\mathrm{eEF} 1 \mathrm{~B} \alpha$ and are strongly conserved as chemically different amino acids in EFL: alignment coordinates 266-267 and 272, corresponding to Q249, D250 and G255 in eEF1A; respectively S264, G265, K270 in EFL (Figures 2 and 3A).

In domain III, differentially conserved sites are mostly dispersed and largely exposed. However, two differentially conserved regions are positioned to be involved in the eEF1A:eEF1B $\alpha$ interaction (Figures 2 and 3A): firstly DCHTAHI in eEF1A, which is FVR.GRs in EFL (starting at position 383 in Figure 2, 360 in S. cerevisiae eEF1A and 381 in C. incerta EFL), and secondly amino acids GN in EFL, conserved as MR in eEF1A starting at position 450 in Figure 2, 427 in S. cerevisiae eEF1A and 448 in $C$. incerta EFL). eEF1A is extended in sequence at the extreme $\mathrm{C}$ terminus by an average of 17 amino acids, relative to EFL (Figure 2). The amino acid contacts in this region are unknown as they are not present in the crystal structure.

Mutants of eEF1A in S. cerevisiae have previously been shown to confer independence from eEF1Ba: R164K, T22S, 


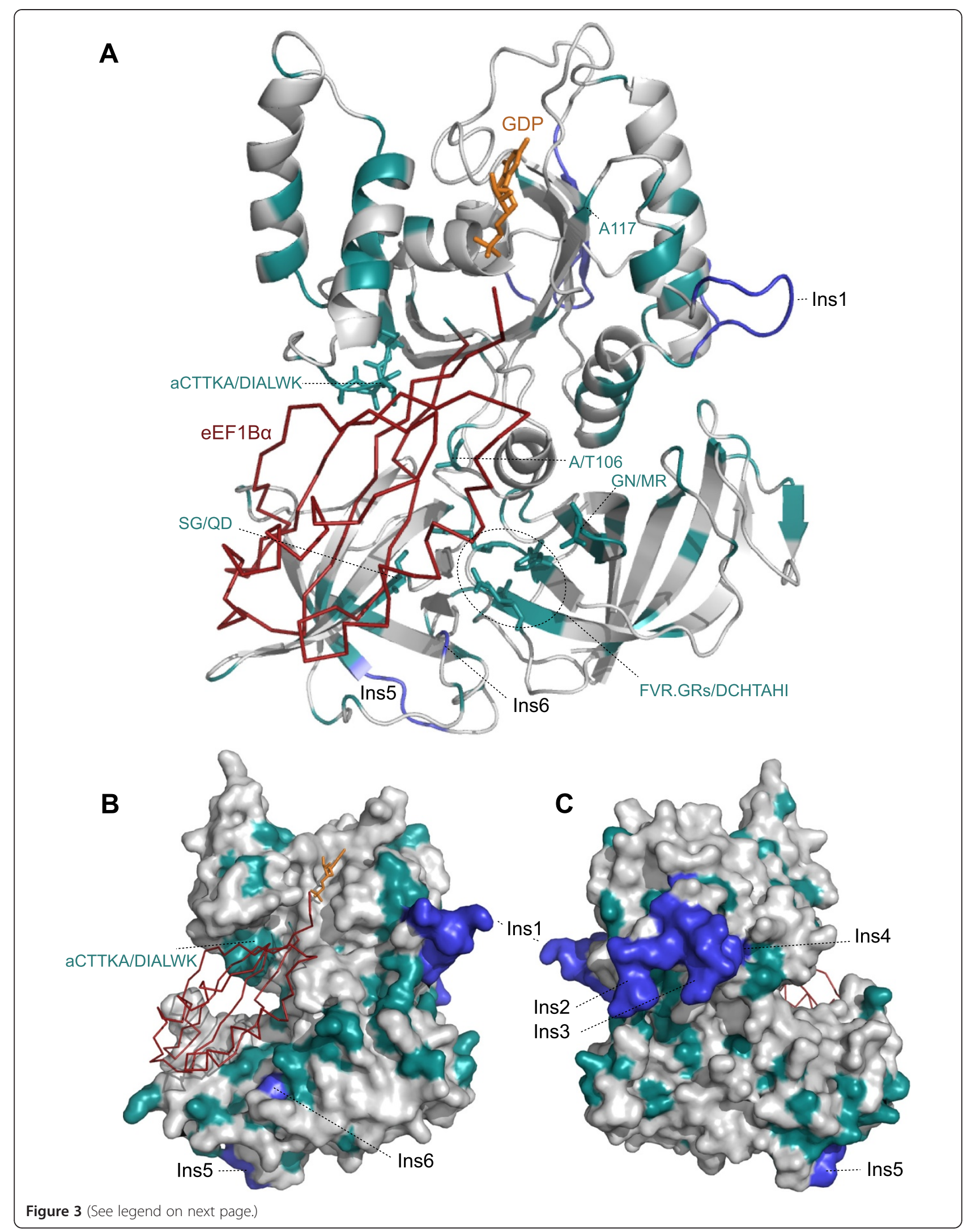


A112T, and A117V [24] (Figure 2). These are mostly located in the G domain and surprisingly, only two differ in conservation between eEF1A and EFL: A112 is unconserved in EFL, and A177 is differentially conserved as P117 in EFL. This suggests there are multiple routes to independence from $\mathrm{eEF} 1 \mathrm{~B} \alpha$, although the single amino acid replacement routes may be highly species-specific in their effectiveness. There have been no cases of natural eEF1B $\alpha$-free eEF1As reported, and our distribution analysis suggests this would be rare.

Biochemical experimentation demonstrating rapid selfrecharging of EFL:GDP with GTP would be the unequivocal proof of our hypothesis that EFL can functionally substitute for eEF1A and eEF1B $\alpha$ loss. However, all our attempts to overexpress EFL in E. coli have failed (data not shown). An alternative strategy is to perform in vivo complementation experiments by replacing either both eEF1A and eEF1B $\alpha$ or just eEF1B $\alpha$ with EFL. Using $S$. cerevisiae as a model organism, we generated strains with controlled expression of eEF1A, eEF1B $\alpha$ and M. brevicollis EFL (see Additional file 3: Materials and methods). Removal of saver plasmids expressing either both $\mathrm{eEF} 1 \mathrm{~B} \alpha-$ or eEF1A or just eEF1B $\alpha$ by addition of 5-Fluoroorotic acid (5-FoA) resulted in loss of viability that was not rescued by expression of M. brevicollis EFL (Additional file 4: Figure S2), indicating that in S. cerevisiae, EFL does not seem to be able to complement the loss of either eEF1B $\alpha$ alone or eEF1B $\alpha$ and both genes encoding eEF1A in S. cerevisiae (TEF1 and TEF2 [25]).

\section{Discussion}

The pattern of presence and absence of EFL, eEF1A and eEF1B $\alpha$ allows us to derive a ratchet-like model for the evolutionary dynamics of elongation factor gain and loss in terms of the viable and inviable fates of different combinations of elongation factors (Figure 4). It is likely is that EFL arose by gene duplication after the last common ancestor of eukaryotes and archaea, which encoded eEF1A and $\mathrm{eEF} 1 \mathrm{~B} \alpha$. Degradation of eEF1A or eEF1B $\alpha$ though random genetic drift in the presence of EFL is likely to be an almost irreversible step that acts as the pawl of the ratchet: a return to the ancestral state would require that the lost gene is quickly re-transferred before its binding partner diverges beyond preventing functional interaction: an unlikely scenario.

The ratchet may also work in the reverse direction, i.e. once EFL is lost from an EFL + eEF1A + eEF1B $\alpha$-encoding organism there is no going back. This depends on how frequently, if at all, EFL is transmitted by HGT, which is currently unclear [12-17]. In the absence of EFL HGT, the ratchet is nonprocessive and acts merely as a lineagesorting evolutionary mechanism, degrading EFL + eEF1A + eEF1B $\alpha$-encoding organisms into either EFL- or eEF1A + eEF1B $\alpha$-encoding. Repetitive re-introduction of EFL by HGT into an eEF1A + eEF1B $\alpha$ background would make the ratchet processive, potentially leading to an enrichment of EFL-containing organisms. Given the uncertainty in EFL HGT rates, it is impossible to assess the processivity of the ratchet. In the most extreme case of nonprocessivity, EFL would not have been subject to HGT at all, would have been present in the eukaryotic ancestor, and then all three genes would have been maintained for millions of years before the divergence of all modern EFL-encoding groups of organisms.

The relative rates of transitioning between states of factor composition is likely to be highly species specific; while EFL is clearly capable of replacing eEF1A in multiple lineages, it has been experimentally shown that Diplonema EFL can be co-maintained with, but can not replace eEF1A in Trypanosoma [18]. Our results suggest the same is true for replacement of either both eEF1A and eEF1B $\alpha$ or just eEF1B $\alpha$ with $M$. brevicollis EFL in S. cerevisiae (Additional file 4: Figure S2). EFL is not naturally found in any yeasts, which may reflect an irreplaceability of yeast eEF1A, or may be because successful HGT is rare in this group of organisms [26].

The stability of the intermediate states (EFL in combination with eEF1A and/or eEF1B $\alpha$ ) depends on organismspecific constraints such as multifunctionalisation (which may drive the system towards co-maintenance of both paralogs), and evolutionary selection for genome reduction (which could increase the rate of loss). The rare cases of dual maintenance may be driven by multifunctionality of eEF1A [12]. In fact it is surprising that eEF1A is not maintained in parallel to EFL more often given its plethora of "moonlighting functions". One explanation could be that eEF1A is not universally multifunctional, or its additional functions do not provide enough selective advantage for its maintenance. It is also possible that some of the moonlighting functions could be carried out by EFL, or by one of the other two closely related paralogs of eEF1A, eRF3 or Hbs1p. Indeed, eRF3 and Hbs1p have already taken over eEF1A's additional ancestral functions in translation termination and mRNA decay via eRF1 and Dom34p binding [27,28]. 


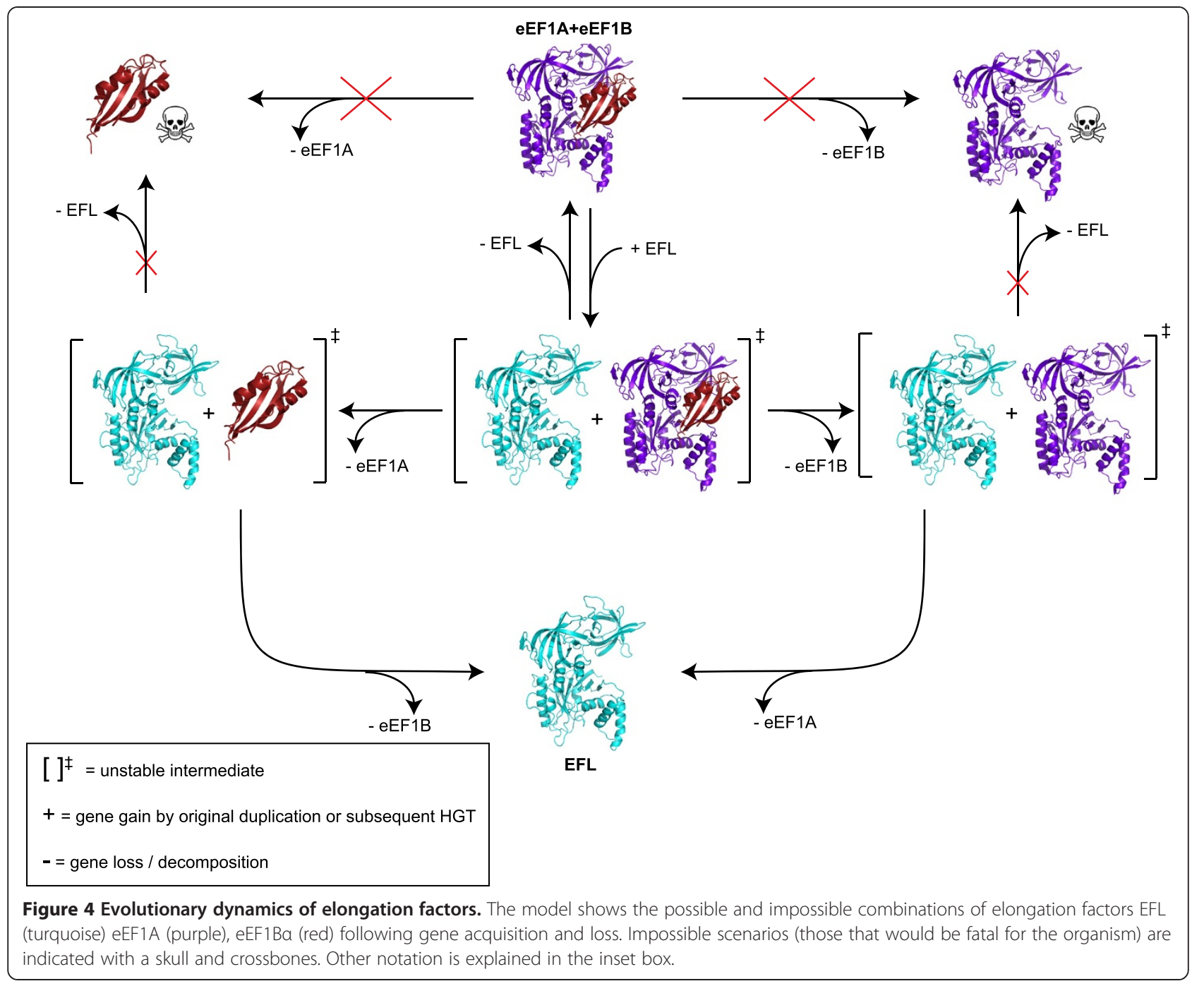

Our sequence conservation and homology modeling analyses indicate several sequence regions that may be responsible for EFL's lack of requirement for eEF1B $\alpha$. However, we can not rule out the possibility that EFL has hijacked another GEF for recharging. One candidate could be eIF2 $\alpha$, the GEF for an ancient paralog of eEF1A and EFL, eIF2X. Although homology is not apparent at the sequence level, eIF $2 \alpha$ is structurally similar to $\mathrm{eEF} 1 \mathrm{~B} \alpha[29]$.

The ratchet mechanism of elongation factor replacement relies only on random genetic drift and can explain how eEF1A can be efficiently replaced by EFL without the need of the latter being a "better" elongation factor, i.e. providing a selective advantage in itself. The ability of EFL to recharge without a specialized accessory factor does not in itself make it an improved enzyme; the impact of this reduction on the functional cycle of the elongation factor is unknown.

\section{Conclusions}

The genomic distribution of the guanine exchange factor eEF1B $\alpha$ considered alongside that of EFL and eEF1A gives a very strong indication that EFL is able to recharge without this exchange factor. Thus, the presence of EFL has apparently allowed the decay and loss of both eEF1A and eEF1B $\alpha$ in some lineages of eukaryotes, a ratchet-like process where return to the ancestral state is unlikely. Horizontal transmission of EFL has been proposed among eukaryotes, however current sequence data is inadequate for determining the rate of transfer, and indeed if it occurs at all. Additional sequencing efforts are required to more fully resolve the dynamics of EFL through the evolutionary history of eukaryotes. Further in vitro and in vivo experimentation is also required to answer the question of whether EFL selfrecharges or whether exchange is promoted by another factor. 


\section{Methods}

BLAST searches were carried out at the JGI (http://genome.jgi.doe.gov/), NCBI (http://ncbi.nlm.nih.gov/), Origin of Multicellularity [30], GeneDB [31] and Cyanidioschyzon merolae genome (http://merolae.biol.s.u-tokyo.ac.jp/) database webpages, using $M$. brevicolis EFL, Monosiga sp. (ATCC 50635) eEF1A and S. cerevisiae eEF1B $\alpha$ as queries. The BLASTp method was used where protein models were available; otherwise tBLASTn was used to search protein against translated genomic and EST nucleotide sequences. Nucleotide hits were translated into protein using Transeq at the EBI (http://www.ebi.ac.uk/Tools/). The $E$ value limit was set to $1 e-5$ and sequences found with $\mathrm{eEF} 1 \mathrm{~B} \alpha$ were checked with Pfam to confirm identity based on the presence of the EF1_GNE (EF1 guanine nucleotide exchange) domain [32]. eEF1B $\alpha$ has been subject to gene duplication in some lineages resulting in paralogs such as eEF1B $\delta$ in Metazoa and eEF1B $\beta$ in plants. As we are interested in presence or absence of a detectable $\mathrm{eEF} 1 \mathrm{~B} \alpha$ homolog and not the complete family tree of this protein family, which has been addressed elsewhere [5], only the top hit was retained. In house translational GTPase datasets (GCA) were used for classification of EFL and eEF1A sequences.

Sequences were aligned using MAFFT v6.864b with the L-ins-i strategy [33]. Consensus sequences were generated with the Consensus Finder Python script [34]. Only full length, non-degrading sequences or identical/nearly identical duplicates from the same organism were included in the data set. The threshold conservation level was set to $70 \%$.

For phylogenetic analyses of eEF1A and EFL, gap-rich ambiguous alignment regions were identified by eye and removed. Extremely truncated sequences typical of ESTs were removed to minimize the amount of missing data. This resulted in dataset dimensions of 462 aligned amino acid positions from 72 sequences for EFL, and 446 positions from 82 sequences for eEF1A. Phylogenetic analyses were carried out with RAxML [35] and MrBayes [36] on the CIPRES Science Gateway v3.2 [37]. MrBayes was run with a mixed model plus the gamma rate distribution, with the program converging on the WAG model (1.0 posterior probability) in the case of EFL, and RTREV (0.99 posterior probability) in the case of eEF1A. Two independent runs of 4 chains were run for 2 million generations, sampling every 1000 generations. A consensus tree was generated after a burn in of 200000 generations. At the end of the runs, the standard deviations of split frequencies (SDSF) were 0.01 in the case of EFL and 0.1 in the case of eEF1A. RAxML was run taking into account the MrBayes model selection, with the WAG + CAT model for EFL and RTREV + CAT model for eEF1A with 100 bootstrap replicates in each case.

A structural homology model of EFL was generated using Swiss-Model [38] with the crystal structure of the
eEF1A:eEF1B $\alpha$ complex (PDB ID 1IJE, [8]) as the structural template. Using MacPyMOL (www.pymol.org), the EFL model was structurally aligned with the crystal structure of EF-Tu on the ribosome (PDB IDs 2WRN and 2WRO) [22] in order to indicate likely ribosome and aa-tRNA binding surfaces.

\section{Additional files}

Additional file 1: Table S1. Presence and absence of EFL eEF1A, eEF1Ba with sequence ID numbers. Sequences are ordered by taxonomy. Paler colours indicate that a sequence is present, but highly divergent.

Additional file 2: Figure S1. Phylogenies of eEF1A and EFL. See Additional file 3 for legend.

Additional file 3: Supplementary Methods, full legends for figures S1 and S2, and Table S2 of strains and plasmids used in the study.

Additional file 4: Figure S2. Both S. cerevisiae double eEF1Ba and eEF1A knock-out and eEF1Ba single knock-out are not complemented in vivo by Monosiga brevicollis EFL. See Additional file 3 for legend.

Competing interests

The authors declare that they have no competing interests.

\section{Authors' contributions}

GCA and MC conceived the bioinformatic part of the project, and performed analyses. AK, PK and VH conceived and designed experiments, AK, AS, IC and PK performed experiments, GCA and VH wrote the paper with contributions from MC, AK, PK, and AS. TT and $\mathrm{VH}$ contributed reagents. All authors read and approved the final manuscript.

\section{Acknowledgments}

We thank Barry Leadbeater for providing M. brevicollis culture, Terry Kinzy for providing S. cerevisiae strains TKY961 and JWY4231 as well as plasmid pJWB2937, and Thomas D Fox for the S. cerevisiae DUL2 strain and experimental advice. This work was supported by the funds from European Regional Development Fund through the Centre of Excellence in Chemical Biology ( $\mathrm{VH}$ and TT), Estonian Science Foundation grants (ETF9012 and PUT37 to VH, ETF9020 to GCA); Swedish Research council and Umeå University (VH); Archimedes Foundation (AK); "U.M.N.I. K" program (AK), Russian Foundation for Basic Research (AK, IC and PK) and European Social Fund grant "Mobilitas" MJD99 (GCA).

\section{Author details}

${ }^{1}$ University of Tartu, Institute of Technology, Nooruse 1, 50411 Tartu, Estonia. 2Department of Molecular Biology, Umeå University, Umeå, Sweden. ${ }^{3}$ Laboratory for Molecular Infection Medicine Sweden (MIMS), Umeå University, Umeå, Sweden. ${ }^{4}$ Department of Molecular Biology, Faculty of Biology, Moscow State University, Moscow, Russia. ${ }^{5}$ School of Applied Sciences, University of Huddersfield, Queensgate, Huddersfield HD1 3DH, UK.

Received: 10 September 2013 Accepted: 17 February 2014 Published: 24 February 2014

\section{References}

1. Riis B, Rattan SI, Clark BF, Merrick WC: Eukaryotic protein elongation factors. Trends Biochem Sci 1990, 15(11):420-424.

2. Thanbichler M, Bock A, Goody RS: Kinetics of the interaction of translation factor SelB from Escherichia coli with guanosine nucleotides and selenocysteine insertion sequence RNA. J Biol Chem 2000, 275(27):20458-20466.

3. Gromadski KB, Schummer T, Stromgaard A, Knudsen CR, Kinzy TG, Rodnina MV: Kinetics of the interactions between yeast elongation factors $1 \mathrm{~A}$ and 1Balpha, guanine nucleotides, and aminoacyl-tRNA. J Biol Chem 2007, 282(49):35629-35637.

4. Gromadski KB, Wieden HJ, Rodnina MV: Kinetic mechanism of elongation factor Ts-catalyzed nucleotide exchange in elongation factor Tu. Biochemistry 2002, 41(1):162-169. 
5. Le Sourd F, Boulben S, Le Bouffant R, Cormier P, Morales J, Belle R, Mulner-Lorillon O: eEF1B: at the dawn of the 21st century. Biochim Biophys Acta 2006, 1759(1-2):13-31.

6. Slobin LI, Moller W: Purification and properties of an elongation factor functionally analogous to bacterial elongation factor Ts from embryos of Artemia salina. Eur J Biochem 1978, 84(1):69-77.

7. Andersen GR, Pedersen L, Valente L, Chatterjee I, Kinzy TG, Kjeldgaard M, Nyborg J: Structural basis for nucleotide exchange and competition with tRNA in the yeast elongation factor complex eEF1A:eEF1Balpha. Mol Cell 2000, 6(5):1261-1266.

8. Andersen GR, Valente L, Pedersen L, Kinzy TG, Nyborg J: Crystal structures of nucleotide exchange intermediates in the eEF1A-eEF1Balpha complex. Nat Struct Biol 2001, 8(6):531-534

9. Sasikumar AN, Perez WB, Kinzy TG: The many roles of the eukaryotic elongation factor 1 complex. Wiley Interdiscip Rev RNA 2012, 3(4):543-555.

10. Visweswaraiah J, Lageix S, Castilho BA, Izotova L, Kinzy TG, Hinnebusch AG, Sattlegger E: Evidence that eukaryotic translation elongation factor $1 \mathrm{~A}$ (eEF1A) binds the $\mathrm{Gcn} 2$ protein $\mathrm{C}$ terminus and inhibits $\mathrm{Gcn} 2$ activity. J Biol Chem 2011, 286(42):36568-36579.

11. Keeling $P$, Inagaki Y: A class of eukaryotic GTPase with a punctate distribution suggesting multiple functional replacements of translation elongation factor 1alpha. Proc Natl Acad Sci U S A 2004, 101(43):15380-15385.

12. Kamikawa R, Brown MW, Nishimura Y, Sako Y, Heiss AA, Yubuki N, Gawryluk R, Simpson AG, Roger AJ, Hashimoto T, Inagaki Y: Parallel re-modeling of EF-1alpha function: divergent EF-1alpha genes co-occur with EFL genes in diverse distantly related eukaryotes. BMC Evol Biol 2013, 13:131.

13. Gile GH, Faktorova D, Castlejohn CA, Burger G, Lang BF, Farmer MA, Lukes J, Keeling PJ: Distribution and phylogeny of EFL and EF-1alpha in Euglenozoa suggest ancestral co-occurrence followed by differential loss. PLOS ONE 2009, 4(4):e5162

14. Ishitani $Y$, Kamikawa R, Yabuki A, Tsuchiya M, Inagaki $Y$, Takishita K. Evolution of elongation factor-like (EFL) protein in Rhizaria is revised by radiolarian EFL gene sequences. J Eukaryot Microbiol 2012, 59(4):367-373.

15. Kamikawa R, Inagaki Y, Sako Y: Direct phylogenetic evidence for lateral transfer of elongation factor-like gene. Proc Natl Acad Sci U S A 2008, 105(19):6965-6969.

16. Kamikawa R, Yabuki A, Nakayama T, Ishida $K$, Hashimoto $T$, Inagaki Y: Cercozoa comprises both EF-1alpha-containing and EFL-containing members. Eur J Protistol 2011, 47(1):24-28.

17. Sakaguchi M, Takishita K, Matsumoto T, Hashimoto T, Inagaki Y: Tracing back EFL gene evolution in the cryptomonads-haptophytes assemblage: separate origins of EFL genes in haptophytes, photosynthetic cryptomonads, and goniomonads. Gene 2009, 441(1-2):126-131.

18. Szabova J, Ruzicka P, Verner Z, Hampl V, Lukes J: Experimental examination of EFL and MATX eukaryotic horizontal gene transfers: coexistence of mutually exclusive transcripts predates functional rescue. Mol Biol Evol 2011, 28(8):2371-2378.

19. Henk DA, Fisher MC: The gut fungus Basidiobolus ranarum has a large genome and different copy numbers of putatively functionally redundant elongation factor genes. PLOS ONE 2012, 7(2):e31268.

20. Kamikawa R, Sakaguchi M, Matsumoto T, Hashimoto T, Inagaki Y: Rooting for the root of elongation factor-like protein phylogeny. Mol Phylogenet Evol 2010, 56(3):1082-1088.

21. Cocquyt E, Verbruggen H, Leliaert F, Zechman FW, Sabbe K, De Clerck O: Gain and loss of elongation factor genes in green algae. BMC Evol Biol 2009, 9:39.

22. Schmeing TM, Voorhees RM, Kelley AC, Gao YG, Murphy FV, Weir JR, Ramakrishnan V: The crystal structure of the ribosome bound to EF-Tu and aminoacyl-tRNA. Science 2009, 326(5953):688-694

23. Baldauf SL, Palmer JD: Animals and fungi are each other's closest relatives: congruent evidence from multiple proteins. Proc Natl Acad Sci U S A 1993, 90(24):11558-11562.

24. Ozturk SB, Kinzy TG: Guanine nucleotide exchange factor independence of the G-protein eEF1A through novel mutant forms and biochemical properties. J Biol Chem 2008, 283(34):23244-23253.

25. Kinzy TG, Woolford JL Jr: Increased expression of Saccharomyces cerevisiae translation elongation factor 1 alpha bypasses the lethality of a TEF5 null allele encoding elongation factor 1 beta. Genetics 1995, 141(2):481-489.

26. Dujon B, Sherman D, Fischer G, Durrens P, Casaregola S, Lafontaine I, De Montigny J, Marck C, Neuveglise C, Talla E, Goffard N, Frangeul L, Aigle M, Anthouard V, Babour A, Barbe V, Barnay S, Blanchin S, Beckerich JM, Beyne E,
Bleykasten C, Boisrame A, Boyer J, Cattolico L, Confanioleri F, De Daruvar A, Despons L, Fabre E, Fairhead C, Ferry-Dumazet H, et al: Genome evolution in yeasts. Nature 2004, 430(6995):35-44

27. Atkinson GC, Baldauf SL, Hauryliuk V: Evolution of nonstop, no-go and nonsense-mediated mRNA decay and their termination factor-derived components. BMC Evol Biol 2008, 8(1):290.

28. Saito K, Kobayashi K, Wada M, Kikuno I, Takusagawa A, Mochizuki M, Uchiumi T, Ishitani R, Nureki O, Ito K: Omnipotent role of archaeal elongation factor 1 alpha (EF1alpha in translational elongation and termination, and quality control of protein synthesis. Proc Natl Acad Sci U S A 2010, 107(45):19242-19247.

29. Ito T, Marintchev A, Wagner G: Solution structure of human initiation factor elF2alpha reveals homology to the elongation factor eEF1B. Structure 2004, 12(9):1693-1704.

30. Ruiz-Trillo I, Burger G, Holland PW, King N, Lang BF, Roger AJ, Gray MW: The origins of multicellularity: a multi-taxon genome initiative. Trends Genet 2007, 23(3):113-118.

31. Hertz-Fowler C, Peacock CS, Wood V, Aslett M, Kerhornou A, Mooney P, Tivey A, Berriman M, Hall N, Rutherford K, Parkhill J, Ivens AC, Rajandream MA, Barrell B: GeneDB: a resource for prokaryotic and eukaryotic organisms. Nucleic Acids Res 2004, 32(Database issue):D339-D343.

32. Finn RD, Mistry J, Tate J, Coggill P, Heger A, Pollington JE, Gavin OL, Gunasekaran P, Ceric G, Forslund K, Holm L, Sonnhammer EL, Eddy SR, Bateman A: The Pfam protein families database. Nucleic Acids Res 2010, 38(Database issue):D211-D222.

33. Katoh $\mathrm{K}$, Kuma $\mathrm{K}$, Toh $\mathrm{H}$, Miyata T: MAFFT version 5: improvement in accuracy of multiple sequence alignment. Nucleic Acids Res 2005 33(2):511-518.

34. Atkinson GC, Baldauf SL: Evolution of elongation factor $\mathrm{G}$ and the origins of mitochondrial and chloroplast forms. Mol Biol Evol 2011, 28(3):1281-1292.

35. Stamatakis A: RAxML-VI-HPC: maximum likelihood-based phylogenetic analyses with thousands of taxa and mixed models. Bioinformatics 2006, 22(21):2688-2690.

36. Huelsenbeck JP, Ronquist F: MRBAYES: Bayesian inference of phylogenetic trees. Bioinformatics 2001, 17(8):754-755.

37. Miller MA, Pfeiffer W, Schwartz T: Creating the CIPRES Science Gateway for inference of large phylogenetic trees. New Orleans, LA: Gateway Computing Environments Workshop (GCE): 2010; 2010:1-8.

38. Arnold K, Bordoli L, Kopp J, Schwede T: The SWISS-MODEL workspace: a web-based environment for protein structure homology modelling. Bioinformatics 2006, 22(2):195-201.

doi:10.1186/1471-2148-14-35

Cite this article as: Atkinson et al: An evolutionary ratchet leading to loss of elongation factors in eukaryotes. BMC Evolutionary Biology 2014 14:35.

\section{Submit your next manuscript to BioMed Central and take full advantage of:}

- Convenient online submission

- Thorough peer review

- No space constraints or color figure charges

- Immediate publication on acceptance

- Inclusion in PubMed, CAS, Scopus and Google Scholar

- Research which is freely available for redistribution 